the reading of the Romantic writers such as Samuel Taylor Coleridge. The conclusion almost wistfully points to the end of an optimistic world and the onset of the romantic period that "spurned the arid insights of reason and the denial of innate instincts". In a sense, therefore, Uglow, who is honorary professor of English and comparative literature at Warwick University, is in a specific dialogue with readers of the Romantics, urging them to look to the context of the Lunar Men and their concerns.

The specificity of the audience points perhaps to a weakness in the book. It will be enjoyed by many even if they do not pick up every allusion, but scientists may be surprised that the author does not put the Lunar Society into its context in scientific history. The Lunar Men were succeeded not just by the Romantic poets, but also by the British Association for the Advancement of Science, founded in 1831 with such a diverse membership of enthusiasts that William Whewell felt compelled to invent a new name to describe them: "scientists".

Moreover, although the technological meaning of science is well covered - one might even say exaggerated - its cultural function of aiding understanding of a new, complex, urban, multi-ethnic, multi-class world is neglected. What was the place of the Lunar Men as a group in the Industrial Revolution? Uglow will imply their centrality, but does not decisively address the point. So could the book be better? Certainly many of its readers will go away with unanswered questions and a wish to argue with the author. But that is to attest to achievement, not failure.

Robert Bud is at the Science Museum,

Exhibition Road, London SW7 2DD, UK.

\section{Einstein brought up to speed}

The Curvature of Spacetime: Newton, Einstein, and Gravitation by Harald Fritzsch

Columbia University Press: 2002. 341 pp. \$29.95, £20

\section{Francis Everitt}

When Daniel Defoe's fictional castaway Robinson Crusoe finally collected his thoughts on 30 September 1659, on the lonely island where he would remain for 28 years, 2 months and 19 days before returning to England in 1687, the year Newton's Principia was published, his first action was to write down in the notebook he had providentially saved from the wreckage a debit-credit account of his state in two columns, Evil and Good. Physicists on their island, no less remote, despite being linked

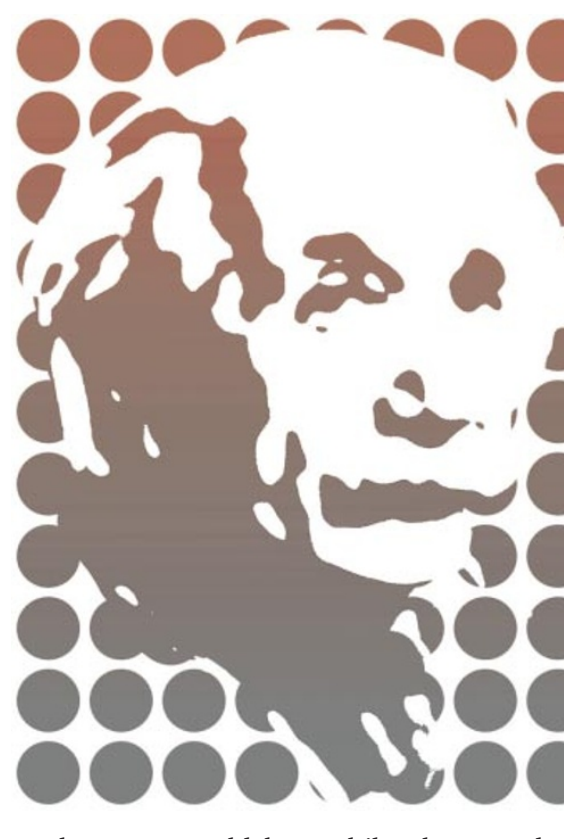

to the outer world by mobile phone and Internet, might well set down a similar debit-credit sheet. Knowing so much, they might start with just one column, headed Good, but eventually comes the need to list in an Evil column a few of the many things we don't understand.

Harald Fritzsch's The Curvature of Spacetime is a time-travel dialogue set in 1996 between three men: Isaac Newton, aged 45 after completing the Principia; Albert Einstein at 51, riding the triumph of relativity; and an imaginary modern expert, Adrian Haller. They meet in various German locations familiar to Einstein, and then at Caltech, where Einstein had spent time in the 1930s. As an expository device, the dialogue form is quite successful. It lets Haller teach Einstein and Newton (and us) the current status, experimental and theoretical, of particle physics, drawing the reader into exchanges of view and conflicting ideas more readily than conventional exposition would allow.

The format is problematic historically, however. We find that Newton apparently knows everything about physics up to about 1890 , yet is fogged and slow on the uptake about Einstein's work. Newton had faults Fritzsch deliberately passes over certain 'disagreeable' aspects of his character — but slowness of uptake was not one of them. And is it pedantry to object to Haller's "reminding" Einstein (in a book containing no mention of Ernest Rutherford) that in 1896 "Henri Becquerel found out that the atomic nucleus of uranium is unstable"? This must have been quite a feat before $\alpha$-particles, $\beta$-particles and $\gamma$-rays were known, the electron was discovered, or Rutherford had proposed the nucleus.

Taken literally, Fritzsch's title is misleading. Much of the book is about particle

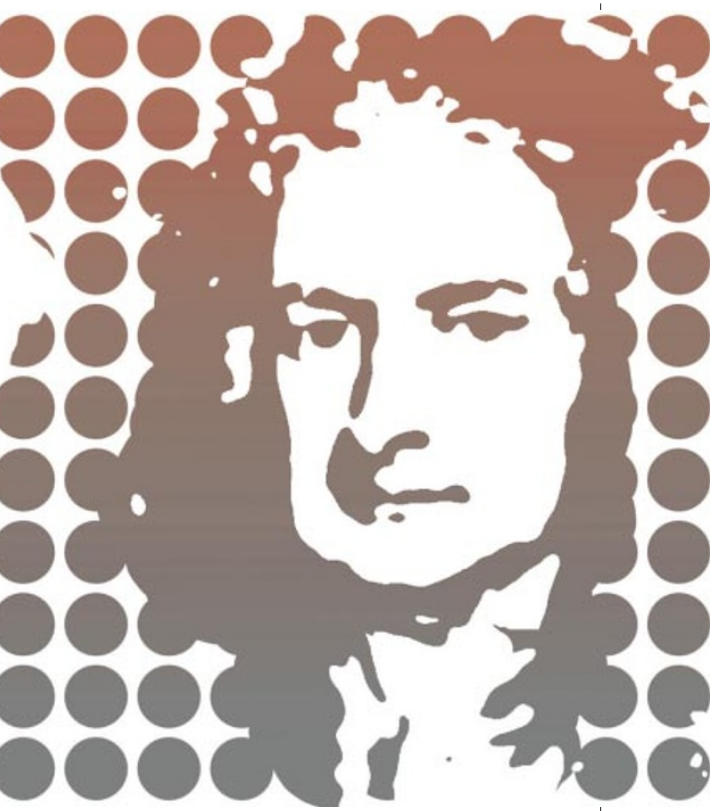

physics, rather than gravity or spacetime. However, this expansion benefits the content; it reveals how far physics has come since Einstein and shows off Fritzsch's considerable gifts as an expositor.

Both Newton and Einstein puzzled over the meaning of mass. Particle physics has failed to illuminate them: the large unexplained range of masses of elementary particles from neutrinos to quarks has only complicated matters still further. Reading Fritzsch on these issues, including his account through Haller of how the Large Hadron Collider at CERN, the European Laboratory for Particle Physics, will (we hope) reveal the Higgs particle and explain mass, leads to the deeper question of what constitutes explanation in physics.

One of Newton's discoveries, often wrongly credited to Galileo, was that in physics, mass fulfils two functions. According to the law of acceleration, mass is the receptacle of inertia; the inverse-square law of gravity makes it the source of gravitation. The masses in these equations are said to be 'equivalent'. Equivalence is often said to be incomprehensible in Newton's physics but explained in Einstein's. Einstein's falling elevator made inertial and gravitational accelerations indistinguishable; this indistinguishability became a principle; curved spacetime followed. And so, Fritzsch has Einstein saying that gravitational and inertial accelerations are not only proportional to each other, but "completely identical".

But surely something is wrong. Complete identity means, in ordinary language, no difference. If there is no difference between inertia and gravity, why does physics need a gravitational constant? Einstein is credited with removing unnecessary concepts he eliminated the ether, and consolidated 
accelerations. His true gift, however, was the unexpected extension of concepts - mass turned into mass-energy, and equivalence became a principle that applies to other phenomena besides mass. His last statement on mass leaves Newton's mystery intact. Distinguishing 'inert mass' from 'heavy mass', Einstein wrote in Out of my Later Years (Philosophical Library, 1950): "That these two radically different definitions lead to the same value for the mass of a body is, in itself, an astonishing fact."

Fritzsch's narrative centres on Einstein. Each chapter head has a quotation from Einstein, and Newton rather improbably seems in awe of him. To find Einstein's true greatness requires an act of distancing. Examining the 23 chapter quotations as an entity shows Einstein not to be necessarily all wise, but certainly to be a most unusual person. Consider this: "All I really want is to sit back and find out how God created this world. It is His thought that I am trying to understand - not the spectral lines of this or that element. I really could not care less about things like those." One may disagree. Great discoveries can come from tiny discrepancies - witness the Lamb shift and quantum electrodynamics. But it is hard to think of anyone but Einstein who would have said that.

Francis Everitt is at the W. W. Hansen

Experimental Physics Laboratory, Stanford

University, Stanford, California 94305, USA.

\title{
•................... A race through the dark
}

\author{
The Extravagant Universe: \\ Exploding Stars, Dark Energy \\ and the Accelerating Cosmos \\ by Robert P. Kirshner \\ Princeton University Press: 2002. 320 pp. \\ $\$ 29.95, £ 19.95$

\section{Sean Carroll}

The 1990s will go down in history as the decade in which we successfully inventoried the constituents of the Universe. A series of ground-breaking observations have given us a picture in which ordinary matter - atoms, stars, gas and dust - accounts for only about $5 \%$ of the total energy of the cosmos, with $25 \%$ consisting of 'dark matter', which is different from any of the known elementary particles. Most surprising of all, fully $70 \%$ of the Universe is the utterly mysterious 'dark energy', characterized by the fact that it is distributed nearly uniformly through space and evolves very slowly (if at all) with time.

The leading candidate for dark energy is Einstein's cosmological constant, which is equivalent to a non-zero minimum amount of energy density at every point in space.

Even though we have measured the amounts of dark matter and dark energy, and can describe some of their simple properties, we know next to nothing about their origin and nature, and are worse than clueless about why they are as abundant as they are. So there is still important work to be done.

Robert Kirshner played a major role in the discovery that the Universe is accelerating, thereby providing the first direct evidence for the existence of dark energy. In a Universe dominated by ordinary matter, the expansion rate would slow down with time; in contrast, dark energy has a negative pressure, the gravitational effect of which is to make the recession velocity of distant galaxies appear to speed up.

In The Extravagant Universe, Kirshner tells the story of how astronomers figured out how to use type Ia supernovae as "standardizable candles" to accurately measure huge distances, and then performed largescale searches for such objects to determine the behaviour of the Universe's expansion as a function of time. The Extravagant Universe is a personal book, rather than an objective account, intermingling the history of cosmology with an explanation of supernovae through the story of Kirshner's own research on these unique celestial events. For the general reader interested in the excitement of how science is done, this strategy makes for a fascinating account. Although several books by leading physicists have recently mixed autobiography with scientific exposition, most have been by theorists. It is refreshing to get the perspective of someone who has to organize his calendar around the phases of the Moon (in order to leave free those nights when the skies are darkest and best for observation).

Of course, writing a book from a personal viewpoint frees an author from the responsibility of devoting equal amounts of time to the work of all competing groups, although one must still be fair. After a pioneering but premature effort in the 1980s by a group of Danish astronomers, the epochal discovery of universal acceleration in 1998 was the work of two competing teams: the High-Z Supernova Search Team led by Brian Schmidt (of which Kirshner, who was Schmidt's thesis adviser, is a member), and the Supernova Cosmology Project led by Saul Perlmutter. In this mostly friendly rivalry, there is inevitably some jockeying for position in the apportionment of credit; it seems clear, however, that posterity will give both groups full credit for discovering dark energy.

Kirshner is a talented writer, and both experts and general readers will find his book a consistently enjoyable read. He takes the time to tell delightful and surprising stories about the many personalities who have contributed to modern cosmology. You will learn, for example, that the first man-made satellite to escape Earth's orbit was designed by Caltech astronomer Fritz Zwicky, and launched in 1957. (I won't give

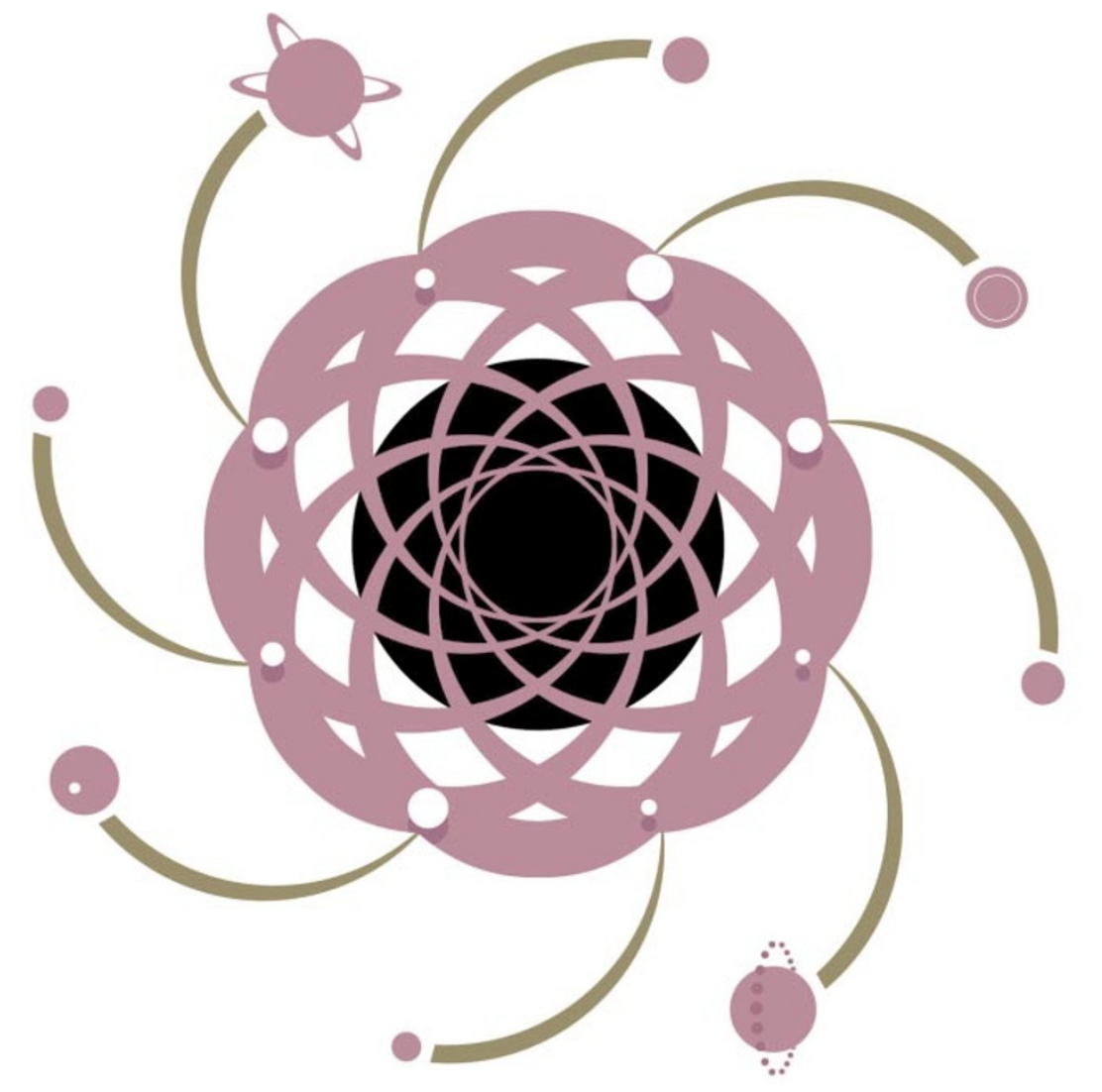

